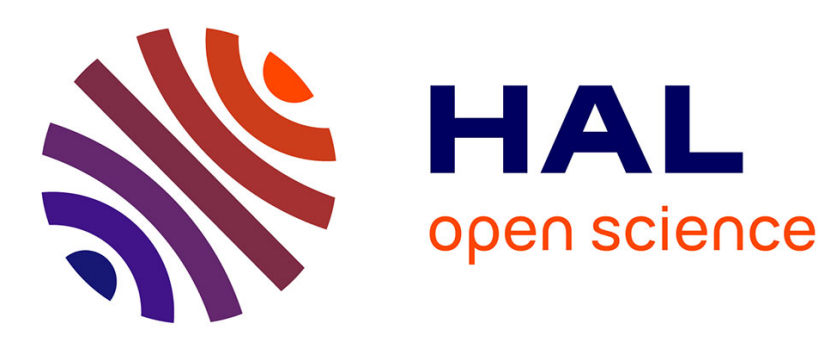

\title{
Characterization of a system generating a homogeneous isotropic turbulence field by free synthetic jets
}

Charles Goepfert, Jean-Louis Marié, Delphine Chareyron, Michel Lance

\section{To cite this version:}

Charles Goepfert, Jean-Louis Marié, Delphine Chareyron, Michel Lance. Characterization of a system generating a homogeneous isotropic turbulence field by free synthetic jets. Experiments in Fluids, 2010, 48, pp.809-822. 10.1007/s00348-009-0768-5 . hal-00566039

\section{HAL Id: hal-00566039 \\ https://hal.science/hal-00566039}

Submitted on 13 Jun 2012

HAL is a multi-disciplinary open access archive for the deposit and dissemination of scientific research documents, whether they are published or not. The documents may come from teaching and research institutions in France or abroad, or from public or private research centers.
L'archive ouverte pluridisciplinaire HAL, est destinée au dépôt et à la diffusion de documents scientifiques de niveau recherche, publiés ou non, émanant des établissements d'enseignement et de recherche français ou étrangers, des laboratoires publics ou privés. 
Exp Fluids (2010) 48:809-822

DOI $10.1007 / \mathrm{s} 00348-009-0768-5$

\section{Characterization of a system generating a homogeneous isotropic turbulence field by free synthetic jets}

Charles Goepfert · Jean-Louis Marié •

Delphine Chareyron - Michel Lance

C. Goepfert · J.-L. Marié - D. Chareyron · M. Lance

Laboratory of Fluid Mechanics and Acoustics UMR CNRS

5509, University of Lyon, Lyon, France

M. Lance

e-mail: michel.lance@ec-lyon.fr

C. Goepfert · J.-L. Marié $(\bowtie)$ - D. Chareyron · M. Lance

Ecole Centrale de Lyon, Université Lyon1, INSA Lyon,

69134 Ecully, France

e-mail: jean-louis.marie@ec-lyon.fr 


\title{
Characterization of a system generating a homogeneous isotropic turbulence field by free synthetic jets.
}

\author{
Charles Goepfert, Jean-Louis Marié, Delphine Chareyron, Michel Lance \\ Laboratory of Fluid Mechanics and Acoustics UMR CNRS 5509, \\ University of Lyon \\ Ecole Centrale de Lyon / Université Lyon1 / INSA Lyon \\ 69131 Ecully, France \\ jean-louis.marie@ec-lyon.fr
}

\begin{abstract}
A facility inspired by Hwang and Eaton (2004) for generating a homogeneous isotropic turbulence was built, the objective being to study evaporating droplets in the presence of turbulence. Turbulence was produced by the mixing of six synthetic jets, in ambient atmosphere. Combined PIV and LDA techniques were used to measure the statistical turbulence properties. The turbulence produced was found to be homogeneous isotropic with a small mean flow within a domain having an average size of $50 \times 50 \times 50 \mathrm{~mm}$. The rms fluctuations were of the order of $0.9 \mathrm{~m} / \mathrm{s}$, corresponding to a Taylor Reynolds number of 240 and an integral length scale of about $40 \mathrm{~mm}$. This apparatus proved to be well suited to the study of the evaporation of droplets in a controlled turbulence field.
\end{abstract}

\section{Introduction}

The concept of homogeneous and isotropic turbulence has proved to be particularly fruitful in the development of statistical theories of turbulence. Many researchers still use it as a powerful model to understand the basic mechanisms underlying more complex turbulent flows, whose analysis is often complicated by the existence of numerous physical couplings. This is for instance the case for flows in the presence of combustion, or two-phase phenomena. The simple framework offered by homogeneous and isotropic turbulence makes it possible to tackle these situations both experimentally and numerically. While Direct Numerical Simulations (DNS) now provide a very accurate and detailed description of turbulent phenomena, experiments still remain unchallenged in the domain of high Reynolds number flows. This is one of the reasons for this study, whose aim is to develop and test an experimental set-up to produce a quasi-isotropic turbulent field in order to investigate the dispersion of evaporating liquid droplets. A number of methods have been proposed in the literature to produce homogeneous turbulent flows as close as possible to this ideal situation. Roughly speaking, most of them fall into two categories: those producing spatially decaying turbulence conveyed at a mean velocity, and those generating stationary turbulence in a box or a tank with zero mean velocity. 
All the experiments with grids in wind or water tunnels like the well-known works of ComteBellot and Corrsin (1966) or Uberoi and Wallis (1967) belong to the first category. In addition to the longitudinal non-homogeneity associated with the decay of turbulent energy, the turbulence fluctuations and the range of turbulent Reynolds numbers remain generally small at usual lab-scale wind tunnel velocities. The use of active grids instead of passive grids significantly increases the level of turbulence produced, at the cost of a loss in isotropy. The use of an oscillating grid in a tank is the most widely used method of the second category (De Silva and Fernando 1994). Turbulence fluctuations thus generated remain small (of the order of $0.1 \mathrm{~m} / \mathrm{s}$ ), but a fairly good degree of isotropy is reached without the need for a long development length. Such systems are used to produce spatially decaying turbulence under sustained oscillations and sometimes temporally decaying turbulence by stopping the forcing. It is worthwhile noting that high Reynolds numbers can be reached using counter-rotating disks in liquid to produce a Von Karman flow (Douady et al. 1991), counter-rotating cylinders in a square tank (Friedman and Katz 2002). The advantage of these devices lies in the higher turbulence levels obtained, but the domain of homogeneity remains somewhat limited. Very recently Variano and Cohen (2008) employed a planar array of randomly actuated synthetic jets disposed on the bottom of a tank. This creates turbulence with a large Reynolds number, an excellent two-dimensional homogeneity and isotropy over a large region, which is well adapted for the study of turbulence at boundaries.

The search for high-efficiency mixing devices adapted to the study of premixed combustion has given rise to several interesting alternative methods. Among them, the systems using fans in the corners of a cubic box, all facing the centre, like in Birouk et al. (1996) are worth noting. Velocity fluctuations as high as $1 \mathrm{~m} / \mathrm{s}$ can be obtained, as well as turbulent Reynolds numbers higher than a few hundred. More recently, Hwang and Eaton (2004a) designed a very simple and elegant method to produce high gas velocity fluctuations at reasonably high Reynolds numbers, while insuring spatial homogeneity and making it possible to perform Lagrangian and Eulerian measurements. The principle of such a facility improved the initial idea of Birouk et al. (1996) by substituting loudspeakers for the eight variable speed fans initially used by these authors. The objective was to study turbulence attenuation by solid particles in zero gravity conditions, which required a compact, robust apparatus, and where there was no risk of moving mechanical parts breaking down (Hwang and Eaton 2004b). Like in Birouk et al. (1996), loudspeakers were fixed in the corners of a cubic chamber. The membranes of the loudspeakers pushed air out of a plenum through an orifice and generated eight synthetic jets producing very strong turbulence at the centre of the chamber. Isotropic stationary or decaying turbulence was thus obtained within a homogeneity domain of $40 \times 40$ 
$\times 40 \mathrm{~mm}$. The zero gravity conditions required the use of a box with solid walls, which introduced some confinement outside the domain where quasi-isotropy was met. In particular, Hwang and Eaton (2004b) noted the existence of a secondary motion in the chamber which generally remained weak, but could under certain conditions be more pronounced (Hwang and Eaton (2001), Hwang and Eaton (2006)). This motion was responsible for a small vertical mean flow inside the domain, typically equal to $10 \%$ of the r.m.s velocities and less than $30 \%$ of the r.m.s velocities in the less favourable case. As long as turbulence modulation by solid particles is concerned, like in Hwang and Eaton (2006), this effect is not disruptive for the study. This might not be the case for other situations such as the one mentioned before, where Lagrangian tracking of the evaporating droplets is required. Indeed, the main problem in this case arises from droplets that leave the quasi-isotropic domain, might be transported outside, and due any possible recirculation generated by the confinement, be re-injected into the isotropic turbulence during their evaporating process. Moreover, the presence of the walls complicates the introduction of droplets into the domain of interest of the turbulent field and makes the use of optical methods more difficult due to the possible formation of a liquid film. In this paper we extend the original idea of Hwang and Eaton to a non-confined facility, which is an attempt to improve their initial system for use in normal gravity conditions and in the presence of phase-change.

In the first section of this paper we describe the apparatus and the measuring techniques. Then we present the characterization of the stationary turbulence field generated in the open facility. The last paragraph is devoted to the results obtained in naturally decaying conditions.

\section{Experimental setup and measuring techniques}

\subsection{Experimental facility}

The configuration adopted here consists of an open "turbulence chamber" without any solid walls, displayed in Fig1a. The loudspeakers are mounted on a cubic frame $\left(0.9 \mathrm{~m}^{3}\right)$ made by the assembly of modular aluminium rods, and pointed towards the centre. Each loudspeaker is glued with a heat conducting silicone mastic onto an aluminium rod, which acts as a cooling device and ensures good thermal stability in time. Two basic geometries can be implemented: one with 8 loudspeakers mounted on the corners of the frame, like in the Hwang and Eaton turbulence chamber, one with 6 loudspeakers mounted on the lateral sides so as to form the vertices of a regular octahedron. It is also possible to work with all 14 loudspeakers arranged thus. With such a system, loudspeakers of different characteristics and sizes can easily be tested and the distance in between varied. The wide optical access resulting from the absence of walls allows velocity and droplet size measurements by using various simultaneous non- 
invasive techniques such as LDA, PIV, PDA, IPI (Interferometric Particle Imaging) or Numerical Holography. In particular, the development of the jets at the outlet of the loudspeakers and in the direction joining two loudspeakers can be investigated, which is more difficult in a closed chamber. The facility is operated in a large room, at ambient pressure and temperature, which minimizes the effect of confinement and therefore the re-circulations induced by the entrainment of air associated with the jets. Pressure and ambient temperature don't need to be precisely controlled to obtain flow repeatability, as far as the balancing procedure of the loudspeakers described thereafter is used.

The set-up with 6 loudspeakers (Fig1a) has been adopted for the present study, because it is simpler to drive. To obtain as large a homogeneous and isotropic domain as possible, the size of the synthetic jets has been maximized by using loudspeakers with large diameter membranes, closed by flat caps with multi-ejection orifices, a large distance apart $(680 \mathrm{~mm})$. The loudspeakers selected for preliminary tests are woofers with coated paper cones manufactured by SEAS (CA21REX: $200 \mathrm{~mm}$ in diameter, 80Wrms, free air resonance frequency $31 \mathrm{~Hz}$ ). They have been then replaced by similar woofers with Carbone membrane manufactured by Davis Acoustics (20 MC8A: 200mm in diameter, free air resonance frequency $37 \mathrm{~Hz}$ ), which prove mechanically more resistant and waterproof. This latter point is crucial when working with droplets and smoke particles for LDA measurements. Membranes push air out of the cavities formed by the caps through a network of 55 round holes, $6 \mathrm{~mm}$ in diameter, arranged in a triangular mesh $20 \mathrm{~mm}$ in size, covering an area $160 \mathrm{~mm}$ in diameter Fig.2. The pulsed jets thus generated merge rapidly and create large powerful turbulent jets at the centre of the frame. This holes network on the caps is chosen to provide both quite high outlet velocities and a uniform momentum flux over the major part of the membranes, without introducing too high a pressure drop which could affect their efficiency over time. Each woofer is driven with sine waves with the same $42 \mathrm{~Hz}$ frequency and phase, generated by a digital/analogical card (National Instrument PCI-6713) in a Labview environment. Driving with a frequency close to the resonance frequency ensures high membrane displacement, for a lower power supply and thus limited heating. Hwang and Eaton used sine waves with random frequencies and phases to avoid the formation of standing waves or other periodic structures within their chamber. This method did not prove to be necessary here with an open facility. The six signals are amplified by three standard 2-channel audio amplifiers. The loudspeakers never produce jets with exactly the same velocity when they are supplied with the same r.m.s voltage. The balance between the 6 jets at the centre of the domain is therefore optimized by finely adjusting these voltages at the beginning of each experiment. This is checked using the LDA technique described hereafter. The balance 
procedure starts when loudspeakers have reached a thermal stability that is after about $60 \mathrm{~min}$. functioning. The balance is achieved for each pair of loudspeakers facing each other, separately, and then for all the pairs together, so as to obtain well symmetrical velocity PDFs, zero mean velocities and equal fluctuations in the $X$ and $Y$ directions. This step can be long and delicate the first time that the loudspeakers are balanced, but the functioning point thus found can be rather easily recovered afterwards. When the loudspeakers are correctly working, the functioning point is generally obtained for r.m.s voltages which are close to each other. Once this is done, the loudspeaker heating being limited, the jets prove to be stable over time typically $60 \mathrm{~min}$. and no further adjustment is thus necessary while running the experiment over such a length of time.

\subsection{PIV measurements and data processing}

Two-dimensional two-component instantaneous turbulent velocity fields are obtained by PIV using a Dantec system including a transmitting arm with light sheet optic and a Flow Map 1500 processor. Two coupled YAG laser sources $(2 \times 120 \mathrm{~mJ}$ Quantel lasers $)$ provide pairs of laser pulses at a frequency of about $10 \mathrm{~Hz}$. The laser light sheet is $0.3 \mathrm{~mm}$ in width. The jets are seeded with micrometer-sized droplets generated by a smoke generator. Seeding is carried out in the surrounding fluid while the woofers are running. Measurements start once the tracers have uniformly diffused all over the working chamber. The light scattered by the tracers illuminated by laser pulses is recorded with a Dantec FlowSense camera (1600 x 1186 pixels, 10 bits). Image pairs are processed according to the adaptive correlation method. The interrogation window is fixed at $16 \times 16$ pixels with $50 \%$ overlap, resulting in instantaneous velocity fields with $159 \times 127$ vectors. The main planes of view that have been investigated are presented in Fig.1b, with the system of coordinates. The origin $(X=Y=Z=0)$ is taken at the centre of the octahedron formed by the loudspeakers. The plane labelled P1 $(130 \mathrm{~mm}$ $\mathrm{x} 103 \mathrm{~mm}$ ) has been chosen to visualize the flow behind the speakers and to characterize the synthetic jet produced. Planes P2 $(162.1 \times 218.7 \mathrm{~mm}$, spatial resolution $2 \mathrm{~mm} \times 2 \mathrm{~mm})$ and P3 $(62.3 \times 46.2 \mathrm{~mm}$, spatial resolution $0.6 \mathrm{~mm} \times 0.6 \mathrm{~mm})$ are used to determine the limits of the domain of interest and characterize the turbulence field inside. All these planes are located at $Z=0$. The mean flow maps and turbulence characteristics presented in the next section is computed by ensemble averaging over 5,000 instantaneous velocity vector fields. To study the average time evolution of the ring vortices behind the speakers (Plane P1) phase-average velocity fields are obtained by averaging 1000 instantaneous velocity fields, for a given phase in the injection cycle. The whole cycle ("blowing" and "suction") is covered by 16 equally spaced phases. The turbulence domain is also characterized in planes $Z=20 \mathrm{~mm}$ and $Z=40$ 
$\mathrm{mm}$ and in a plane with an angle of $35^{\circ}$, formed by the $X$-axis and the line joining the centres of opposite faces of the octahedron.

\subsection{LDA measurements}

The turbulence characterization performed with PIV was confirmed and completed by 1-point 2-component and 2-point LDA spatial explorations. The emitting optic is a Dantec system including a beam expander (expansion ratio: 1.94), mounted on a three-dimensional displacement system. The light source is a 5W argon-ion laser (Coherent, Innova70) emitting beams of diameter $2.2 \mathrm{~mm}$, at two wave lengths $(514.5 \mathrm{~nm}$ and $488.0 \mathrm{~nm})$. The beam separation at the output of the optics and the focal length are respectively $58.3 \mathrm{~mm}$ and $310 \mathrm{~mm}$ for the 1-point measurements, and $41.3 \mathrm{~mm}$ and $600 \mathrm{~mm}$ for the 2-point measurements. The 2-point measurement method is described in detail in Belmabrouk and Michard (1998) and consists in generating two probe volumes, the first one being kept fixed, while the second one is placed at various separation distances thanks to the rotation of a mirror. The integral length scale is deduced from the transverse velocity correlation function thus measured. The flow is seeded with the same tracers and following the same procedure as for PIV. Doppler signals are processed in coincidence by a Dantec system (57N20 and 57N35 BSA). The mean flow velocities and turbulence characteristics are determined from ensemble-averages over 1,000000 samples, processed with the transit time weighting method proposed by George (1978). This correction proves to be necessary in order to remove the velocity bias likely to occur in the domain of interest, where the mean velocities are small and fluctuations large. This method is efficient when the sampling time is much smaller than the eddy turnover time of the turbulence (Eq.8), which is the case here.

\section{Results}

\subsection{Development of synthetic jets}

The use of perforated caps to increase the spreading of synthetic jets was inspired from the technique of multiple jets found in practical applications such as, multi-jet burners, liquid propellant rocket engines or agricultural sprays. This technique is not common for synthetic jets and to our knowledge, no extensive study has been reported on the subject. The results available in the literature mainly concern the formation and development of single synthetic jets (Glezer and Amitay 2002, Bera et al. 2001), their use as actuators for flow control, and more rarely the interaction of 2 adjacent jets (Smith and Glezer 1998). In the present experiment, the geometrical configuration of the triangular network of pulsed jets described 
above is very close to that of the co-flowing jets investigated by Villermaux and Hopfinger (1994). A sample of the mean velocity field obtained in our working conditions (driving frequency $42 \mathrm{~Hz}$, applied rms voltage 10 volts) in the P1 plane located behind the network is

presented in Fig.3. Symbol ( ) denotes the local ensemble average over all the images. The peak velocity of the jets originating from each hole is equal to $5 \mathrm{~m} / \mathrm{s}$, corresponding to a Reynolds number based on d of 2000. These elementary jets expand with distance from the cap to merge with their neighbours and form a single axisymmetric turbulent flow. The merging operates at a distance $L / M$ between 3 and 4 , comparable to that reported by Villermaux and Hopfinger (1994) at this Reynolds number. The centreline velocity $\bar{U}_{m}$ of the resulting single jet at location $L / M=5$ from the holes is $3 \mathrm{~m} / \mathrm{s}$ and the width of the jet where $\bar{U} / \bar{U}_{m}=0.5$ is about $110 \mathrm{~mm}$. This spreading is greater than that which could be obtained with a synthetic jet from a single orifice.

Phase-averaged velocity fields provide the average time evolution of the ring vortices issuing from each hole for various phases of the injection cycle. Figure 4, which corresponds to the beginning of blowing, shows that the released vortices present good axisymmetry and approximately equal strength all over the surface of the perforated cap. However, the direction of emission of the vortices progressively inclines towards the centre of the jet away from the centreline of the network. The velocity fields for the other phases of the injection cycle show that this effect is caused by the surrounding air which is drawn inwards, parallel to the cap, during the second half of the blowing, and towards the holes during suction. This air movement limits the expansion of the jets located on the periphery of the network and is responsible for the flow convergence appearing in Fig.3. Phase average fields confirm that the mixing of vortices takes place at a distance $L / M$ between 3 and 4, which was checked on the temporal energy spectra by the absence of peaks at the release frequency of vortices and its higher harmonics. Before mixing, the vortices are conveyed in the longitudinal direction with an average speed of $5.4 \mathrm{~m} / \mathrm{s}$, which corresponds to a Strouhal number based on the diameter of the hole, of the order of 0.05 .

\subsection{Properties of the stationary turbulence}

The results presented correspond to a turbulence with $R e_{\lambda} \sim 240$, comparable to that documented in detail by Hwang and Eaton (2004). The turbulence levels and the Reynolds number can be adjusted by changing the driving voltage of the subwoofers. Each new setting of the driving voltage requires the six woofers to be re-balanced and the new turbulence conditions to be characterized in detail. In these experiments, the woofers are balanced under 
the control of LDA measurements, performed at the intersecting point of the six jets $(X=Y=$ $Z=O$ ). Increasing the driving voltage increases the Reynolds number and the intensity of fluctuations. Hwang and Eaton (2001) were able to reach $\operatorname{Re}_{\lambda}$ as high as 450 , but noted the poor repeatability of the flow. This fact is confirmed with the present device, which makes it possible to produce highly turbulent Reynolds number fluctuating fields, but the stationarity of the flow proves to be difficult to maintain. In our case, the reason is that working with high voltages causes an important overheating of the loudspeakers and as a result, the balance of the loudspeakers, which is crucial to get a well homogeneous isotropic domain, is not very stable in time. This difficulty could be overcome by installing a more efficient cooling system of the loudspeakers, like it was done in Hwang and Eaton (2006).

The mean velocity fields obtained by PIV with the large field of view (plane P2) are displayed in Fig. 5. The small triangular area visible in the right-hand upper corner of the field of view corresponds to the boundary of the laser sheet. The size of the spatial domain where the mean velocity is close to zero within $\pm 0.2 \mathrm{~m} / \mathrm{s}$ limits is approximately $50 \mathrm{~mm}$ in the vertical $Y$ direction and $80 \mathrm{~mm}$ in the horizontal $X$ direction. The fact that it is smaller along the vertical axis is the consequence of the flow generated by the pair of woofers aligned with this direction (see Fig. 1b). The size along the $Y$ direction is fixed by the depth of mutual penetration of the facing jets and can hardly be extended, even when working with a broad jet cross-section like in this experiment. This phenomenon constitutes one of the main limitations for this type of turbulence production. As a rule, the homogeneity domain is smaller in the three directions joining the subwoofers and subsequently, the domain of interest is not spherical.

A more precise insight into the domain of interest obtained with the small field of view (plane P3) is shown in Fig. 6. It can be checked on the mean flow velocities and the rms fluctuations that the flow globally exhibits good symmetry in the main $X$ and $Y$ directions. Such a result requires a careful balance of the six jets, obtained by adjusting the driving voltages of each subwoofer. The region where the mean velocity is homogeneous and close to zero within \pm $0.1 \mathrm{~m} / \mathrm{s}$ limits, is a roughly rectangular box of $60 \mathrm{~mm}$ in the $X$ direction by $40 \mathrm{~mm}$ in the $Y$ direction. As confirmed on the $\bar{V}$ component, its size in the $Y$ direction is limited by the 2 incoming flows generated by the vertically placed woofers. Inside this "turbulence box", the rms fluctuations $u^{\prime}, v^{\prime}$ are fairly homogeneous in space, with values 0.84 and $0.9 \mathrm{~m} / \mathrm{s}$ respectively.

Maps of the main flow characteristics inside the turbulence box are reported in Fig. 7. The local mean velocities vary between -0.05 and $0.1 \mathrm{~m} / \mathrm{s}$ for $\bar{U}$, and between -0.1 and $0.05 \mathrm{~m} / \mathrm{s}$ 
for $\bar{V}$. The rms fluctuations divided by their spatial average over the domain, designated by $<>$, lie in the 0.94-1.04 range for $u^{\prime}$ and 0.94-1.06 for $v^{\prime}$, indicating a fairly good degree of homogeneity in space. The isotropy ratio $u$ ' $v$ ' has a value comprised between 0.85 and 1.05 , which means that the isotropy requirement is also well satisfied. The map of the kinetic energy calculated by assuming isotropy in the $Z$ direction, and normalized by its spatial average in the whole domain, exhibits the same good homogeneity in space. This assumption was validated a posteriori by LDA measurements in the third direction. The spatially average skewness $\left\langle S_{u}\right\rangle,\left\langle S_{v}\right\rangle$, and flatness $\left\langle F_{u}\right\rangle,\left\langle F_{v}\right\rangle$ were also calculated from the fluctuations maps. They were found to be close to the values typical of a Gaussian distribution, that is, 0 and 3 respectively.

The velocity PDFs for $U$ (horizontal) and $V$ (vertical) velocity components were obtained at all locations by PIV and LDA. A sample of these PDFs, normalized by the local rms velocity, is provided in Fig.8a and compared to those measured by Hwang and Eaton in 2 directions perpendicular to the faces of their box (i-e not-aligned with the speakers). The PDFs presented by these authors (closed symbols) closely follow the same Gaussian profiles (solid line) in these two directions, with skewness $\approx 0$ and flatness $\approx 3$. Our results exhibit a significant difference in that the distribution in the direction of the speaker ( $V$ component) deviates from Gaussian profiles. This tendency is confirmed by the PDFs obtained at the centre of the domain by PIV and LDA (Fig.8b). However, the deviation remains weak. We believe that it is linked to the flow behaviour at the stagnation point and can hardly be avoided with this way of generating a THI.

Two component LDA measurements of mean velocity, rms fluctuations, isotropy ratio $u$ ' $/ v^{\prime}$, correlation cofficient $u v / u^{\prime} v^{\prime}$, skewness and flatness in the vertical direction, which is the most restrictive regarding homogeneity, agree with the results of PIV. They confirm that the domain where the turbulence can be considered as homogeneous isotropic with a high degree of confidence extends in this direction between $-20 \mathrm{~mm}$ and $+20 \mathrm{~mm}$. However, for some parameters, such as the mean flow velocity or the correlation coefficient, $\pm 30 \mathrm{~mm}$ limits a domain, which is still acceptable.

The flow characteristics are compared in Table 1 with those of Hwang and Eaton (2004). Although the two configurations are slightly different ( 6 free jets for the present study, 8 confined jets for these authors), the characteristics of the two turbulences are very close to each other. The horizontal and the vertical components of the mean velocity are slightly more uniform in the present case, the cause probably being the channel balancing procedure. Indeed, the driving signals all have the same amplification in Hwang and Eaton, while here they are set to optimize the balance between the jets. Also, the absence of a higher vertical 
mean flow in our case might be an indication that there is no significant re circulation motion outside the homogeneous domain. The spatially averaged level of turbulence, the skewness $\left.<S_{u}\right\rangle,\left\langle S_{v}\right\rangle$, and flatness $\left\langle F_{u}\right\rangle,\left\langle F_{v}>\right.$ present similar values, which means that these small differences in the mean flow have no effect on the turbulence parameters. The degree of spatial homogeneity is comparable, but within a domain which turns out to be larger in the present work $(60 \mathrm{~mm} \times 40 \mathrm{~mm})$.

Fig.9 provides an example of the one-dimensional turbulent kinetic Eulerian frequency spectrum measured by LDA at the centre of the turbulence domain. For flow without mean velocity, this spectrum cannot be related to the spatial one, because the classical Taylor's frozen turbulence hypothesis linking frequency and wave number domain does not hold. However, Tennekes (1975) proposed a model of the Eulerian frequency spectrum in homogeneous isotropic turbulence without mean flow, by using a variant of Taylor's hypothesis. Tennekes' basic argument is that, at high Reynolds numbers, the small dissipative eddies are randomly advected, or swept, past the observer by the large energy - containing eddies. If the Reynolds number is large enough for there to be a great separation in scales between the frequencies of the energy containing eddies and the frequencies of the small eddies thus advected, then an inertial subrange on the frequency spectrum with a $-5 / 3$ power law can be expected to exist (Tennekes and Lumley 1974). Although this in theory requires $\operatorname{Re}_{\lambda}$ to be very high, the existence of such an inertial subrange has been experimentally confirmed in different works, both by DNS, as in Yeung and Sawford (2001) for $\operatorname{Re}_{\lambda}=140$ and experimentally, as still recently in Variano and Cowen (2008) for $\operatorname{Re}_{\lambda} \sim 314$. Fig. 9 shows that it is also the case here, and that the $-5 / 3$ power law extends over about one decade. This result seems consistent with the value of $\operatorname{Re}_{\lambda}$ that has been estimated in our experiment (Table 2). On the other hand, no peak at the frequency or the harmonics of the subwoofer is detected. This proves that the initial periodic structure of the synthetic jets has totally disappeared.

The longitudinal and transverse one-dimensional spatial energy spectra, respectively $E_{u u}\left(k_{x}\right)$, $E_{v v}\left(k_{y}\right)$ and $E_{u u}\left(k_{y}\right), E_{v v}\left(k_{x}\right)$, have been computed from our PIV data. They have been obtained by performing a Fourier transform of each image line and ensemble averaging over all the image pairs, as described in (Liu et al. 1999, Hwang and Eaton 2004, De Jong et al. 2009). According to Kolmogorov (1941), these spectra in the inertial range can be modelled as

$$
\begin{aligned}
& E_{\alpha \alpha}\left(k_{\alpha}\right)=\frac{18}{55} C(\varepsilon)^{\frac{2}{3}}\left(k_{\alpha}\right)^{-\frac{5}{3}} \\
& E_{\alpha \alpha}\left(k_{\alpha}\right)=\frac{3}{4} E_{\beta \beta}\left(k_{\alpha}\right)
\end{aligned}
$$


where $C$ is a universal constant $\approx 1.5$ (Pope 2000). The spectra, as calculated from our data, are plotted in Fig.10, together with the models. Since PIV data are discretized over a grid with a finite size, larger than the smallest scales of the turbulence eddies, and cover a limited spatial domain (the domain of homogeneity), the largest wave numbers corresponding to the dissipative scales, as the smallest wave numbers corresponding to the integral scales, are not accurately resolved in the spectra (Hwang and Eaton 2004, Variano and Cohen 2008). Despite this important bias, we see in Fig.10 that the collapse between $E_{u u}\left(k_{x}\right)$, and $E_{v v}\left(k_{y}\right)$ is rather satisfactory, confirming the isotropic character of the flow. The relation between the longitudinal and tranverse spectra is rather well verified. Also, the spectra exhibit a $-5 / 3$ power-law decay at the smaller wave numbers, suggesting that an inertial subrange is partially observed. The best match between Kolmogorov's model and the data in that range is obtained for a dissipation $\varepsilon \approx 3.5-4 \mathrm{~m}^{2} \mathrm{~s}^{-2}$.

Hwang and Eaton (2004) calculated in addition the two dimensional spatial energy spectrum from their PIV data and found that it well agreed with Kolmogorov's spectra at the low wave numbers.

Two-point spatial longitudinal $(X$ direction) and lateral ( $Y$ direction) velocity correlations coefficients were measured both by PIV and LDA. They are respectively defined as:

$$
\begin{aligned}
& F_{u u}(r)=R_{u u}^{x}=\overline{u(x, y) u(x+r, y)} / \sqrt{\overline{u(x, y)^{2}}} \sqrt{\overline{u(x+r, y)^{2}}} \\
& F_{v v}(r)=R_{v v}^{y}=\overline{v(x, y) v(x, y+r) /} \sqrt{\overline{v(x, y)^{2}}} \sqrt{\overline{v(x, y+r)^{2}}} \\
& G_{u u}(r)=R_{u u}^{y}=\overline{u(x, y) u(x, y+r)} / \sqrt{\overline{u(x, y)^{2}}} \sqrt{\overline{u(x, y+r)^{2}}} \\
& G_{v v}(r)=R_{v v}^{x}=\overline{v(x, y) v(x+r, y)} / \sqrt{\overline{v(x, y)^{2}}} \sqrt{\overline{v(x+r, y)^{2}}}
\end{aligned}
$$

These correlation coefficients are plotted in Fig.11. In the case of PIV, each coefficient is an average over all pair of points of the homogeneity domain, separated by $r$. In the case of LDA, each coefficient is an average of the values measured by positioning the second probe volume at $\pm \mathrm{r}$ from the centre of the domain. The data obtained by the two methods are in a fairly good agreement, even for separations $r$ where the second LDA probe volume is outside the homogeneity domain ( $\mathrm{r}>20 \mathrm{~mm}$ along the $X$-axis, $\mathrm{r}>30 \mathrm{~mm}$ along the $Y$-axis). Both show that isotropy is best verified for $G$ than for $F$, where a slight difference is noted in the direction aligned with the speakers. Whatever the method, the spatial resolution is not sufficient to calculate the Taylor microscale $\lambda$ from the curvature at the origin. In particular, the 2-point LDA method suffers from the finite size of the two probing volumes (typically $300 \times 50 \mu \mathrm{m})$, which makes the measurement inaccurate when the Taylor length scale is 
smaller than, say, $1 \mathrm{~mm}$. On the other hand, since the two-point correlation curves $F u u, F v v$ don't reach zero, the integral length scale $\Lambda_{f}$ is calculated by fitting a function on these curves using a non-linear least square algorithm. The best fit is obtained with a sum of Gaussians. Integrating the fitting functions yields an integral length scale of the order of $40 \mathrm{~mm}$ for $F u u, 36 \mathrm{~mm}$ for $F v v$, to be compared with the $56 \mathrm{~mm}$ value for Hwang and Eaton. A fit with an exponential is also possible, but overestimates the data for large separations $r$. The relationship between the longitudinal and lateral velocity correlations that are expected from isotropic turbulence theories is written:

$$
G(r)=F(r)+\frac{r}{2} \frac{\partial F(r)}{\partial r}
$$

Fig.11 shows that this relation is well confirmed experimentally, in agreement with the findings.

The main properties of the turbulence are summarized in Table 2 and compared to those of the turbulence box of Hwang and Eaton. Accurate measurement of the viscous dissipation is very difficult, even in isotropic flows, because eddies having a size in the range of the Kolmogorov's scale are spatially filtered by PIV or LDA. So, dissipation and all the properties of the turbulence were first estimated from the turbulent kinetic energy $q^{2}$ and the integral length scale $\Lambda_{f}$ inferred from the data, by using the scaling laws valid in homogeneous isotropic turbulence (Batchelor 1953):

$$
\varepsilon=C_{\varepsilon} \overline{\left(\frac{\left(q^{2} / 3\right)^{3 / 2}}{\Lambda_{f}}\right)}, \tau_{\varepsilon} \cong \frac{\overline{\left(q^{2} / 2\right)}}{\varepsilon}, \tau_{k} \cong \overline{\left(\frac{v}{\varepsilon}\right)^{1 / 2}}, \eta \cong \overline{\left(\frac{v^{3}}{\varepsilon}\right)^{1 / 4}}
$$

$\tau_{\varepsilon}$ denotes the eddy turnover time, $\tau_{k}$ the Kolmogorov time, $\eta$ the Kolmogorov length and $C_{\varepsilon}$ the normalized dissipation rate, which is of order unity. The Taylor microscale $\lambda$ and the associated Reynolds number are given by

$$
\lambda \cong \overline{\left(\frac{5 v q^{2}}{\varepsilon}\right)^{1 / 2}}, \operatorname{Re}_{\lambda} \cong \overline{\left(\frac{\lambda\left(q^{2} / 3\right)^{1 / 2}}{v}\right)}
$$

The universality of the value of $C_{\varepsilon}$ at high Reynolds numbers has been extensively discussed in numerous studies (Sreenivasan 1998, Burattini et al. 2005, Bos et al. 2007). For homogeneous isotropic turbulence kept stationary by injecting energy at the large scales, they show that $C_{\varepsilon}$ is close to 0.5 . Since our flow resembles this situation this value seems to be realistic here. Taking $C_{\varepsilon}$ to be 0.5 yields $\varepsilon=6.7 \mathrm{~m}^{2} / \mathrm{s}^{3}$. With that value, we see in Table 2 that the two turbulences have very similar properties and characteristic scales. It is worth noting 
that Hwang and Eaton determined their characteristics from the turbulent kinetic energy, with the third fluctuation component measured separately by LDA and an estimation of the dissipation calculated from PIV measurements, together with the LES analogy of Sheng et al. (2000) to resolve the small scales of turbulence. In order to check the assumption on the dissipative rate $C_{\varepsilon}, \varepsilon$ has also been estimated from the second-order longitudinal structure function:

$$
\left.D_{v v}(r)=\left(\overline{v(x, y)^{2}-v(x, y+r)^{2}}\right)\right)
$$

computed from our PIV data and spatially averaged over all the domain of homogeneity. Referring to Variano and Cohen (2008) and De Jong et al. (2009), structures functions can be accurately resolved by PIV and do not suffer from the above mentioned spatial filtering which affects the energy spectra. As a result, they appear to be a more robust way for estimating $\varepsilon$ than a simple spectrum fitting. Kolmogorov's second similarity hypothesis (Kolmogorov 1941) implies that, for high Reynolds-number turbulence, in the inertial subrange,

$$
D_{v v}(r)=C_{2}(\varepsilon r)^{\frac{2}{3}}
$$

where the universal constant $C_{2}$ lies in the range 2.0-2.2 (Pope 2000). We see that the secondorder longitudinal structure function, plotted in Fig. 12 under the usual compensated form, exhibits a small plateau in agreement with the theory, from which we deduce $\varepsilon=5.8 \mathrm{~m}^{2} / \mathrm{s}^{3}$ when taking the value $C_{2}=2$. This corresponds to $C_{\varepsilon}$ of the order of 0.44 , which agrees rather well with the above assumption, given the uncertainty on the constant $C_{2}$. The characteristics of the turbulence computed with this second estimation of $\varepsilon$ have been listed in Table2.

\subsection{Decay of a turbulent spot}

Stopping the woofers causes the natural decay of the turbulence. The statistics of this decay were measured using PIV. The procedure was as follows. The woofers were run for approximately 60s, the time needed to reach stationary turbulence, and then turned off, until the turbulence totally vanished and ambient air was at rest. This cycle was repeated one hundred times by driving the signal of the woofers automatically. For each cycle, pairs of images with a time separation of $300 \mu \mathrm{s}$ were taken at time intervals of $0.25 \mathrm{~s}$, starting from $3 \mathrm{~s}$ before the woofers were turned off, and ending $12 \mathrm{~s}$ after. The kinetic energy was then calculated at each of these 60 time steps, from an ensemble average of the 100 velocity fields obtained from the image pairs, assuming isotropy on the third velocity component $W$.

When the forcing by the loudspeakers is stopped, the turbulent energy contained in the 
measurement volume decays under the combined action of viscous dissipation and diffusion. In the absence of a mean velocity field, the balance of kinetic energy density $\frac{\overline{q^{2}}}{2}=\frac{1}{2}\left(\overline{u^{\prime 2}}+\overline{v^{\prime 2}}+\overline{w^{\prime 2}}\right)$, discarding viscous diffusion, is expressed as:

$$
\frac{\overline{\partial q^{2}}}{\partial t}=-\varepsilon-\frac{\partial}{\partial x_{i}}\left(\overline{u_{i}^{\prime} q^{2}}+\frac{\overline{p^{\prime} u_{i}^{\prime}}}{\rho}\right)
$$

where the RHS term is the sum of the dissipation rate $\varepsilon$ and the diffusion by the triple correlation and pressure-velocity correlation, which is significant only at the boundaries of the domain where gradients become important. The latter term acts on the self-diffusion of the turbulent cloud and causes its spatial expansion with time, resulting in a decrease of the local energy density. Therefore, decay for $\overline{q^{2}}$ with time $t$ is expected to be faster than for classical isotropic turbulence such as in Comte-Bellot and Corrsin (1966), Uberoi and Wallis (1967). In the forced turbulence experiments presented above, this non-homogeneous edge effect is also present, but is balanced by the production due to the jets. This faster decay has been observed by Hwang and Eaton (2004a). It confirms that the secondary motion in the chamber is weak and so, quickly dies out after turning out the loudspeakers. However, the decay is not so fast for the case $R e_{\lambda} \sim 415$ documented in Hwang and Eaton (2001), as the secondary mean flow is more pronounced. Due to the absence of confinement, the situation here turns out to represent a slightly different situation, i.e. the evolution of an initially isotropic turbulent "spot" in a quiescent fluid. The time evolution of the total turbulent kinetic energy at the centre of the turbulent box is given in Figs. 13 and 14. In Fig. 14, the ratio of the energy density $\overline{q^{2}}$ at time $t$ to the initial turbulent energy density $\overline{q_{0}^{2}}$ at time $t_{0}$, when the loudspeakers are stopped, is plotted in log-log coordinates versus the time $t$ - $t_{0}$ normalized by the eddy turnover time $\tau_{\varepsilon}$, while Fig. 13 shows a linear plot of the normalized turbulent energy with time from the beginning of the cycle. The slight discontinuities appearing in data in Fig. 13 for times greater than $7 \mathrm{~s}$ arise from the fact that the image pair separation time is kept fixed, equal to $300 \mu \mathrm{s}$. Indeed, the latter, which is well adapted for the beginning of the turbulence decay, becomes too small to achieve particle displacements of a sufficient number of pixels at the end of the decay. As expected, the decay follows a power law $\overline{q^{2}} \propto t^{-n}$, where the exponent $n$ is about $1.6 \pm 0.1$ here. This value is slightly smaller than the one $(n=1.86)$ found by Hwang and Eaton (2004a), but since the fitting of the exponent proves to be sensitive to the value of $t_{0}$, the results are quite consistent in the domain of accuracy. We can conclude that the two situations are very similar and that the decay in the chamber is not 
affected by the confinement effects in that case. Despite the uncertainty on the exponent, it is clear that the decay is faster in the present configuration than in isotropic grid-turbulence, which is commonly observed for diffusive turbulence.

\section{Conclusion}

The original idea proposed by Hwang and Eaton (2004) to generate quasi homogeneous isotropic turbulence in a cubic box has been extended to a non-confined facility, in order to investigate the dispersion of evaporating liquid droplets. Turbulence is produced there, by the mixing of six free synthetic jets working in ambient atmosphere, and set out so they form a regular octahedron. However, a configuration with 8 jets as in these authors or $14(6+8)$ can also be implemented. With this new arrangement, evaporating droplets leaving the turbulence domain will hardly return to it, as it might be the case with the possible secondary motions existing in the box. This is the condition required to study the evaporation process in controlled turbulence.

The turbulent field has been qualified by combining non invasive PIV and LDA techniques for the case $\operatorname{Re}_{\lambda}=240$, which is comparable to the situation documented in detail by these authors. The statistical properties of the confined and non-confined turbulent flows in the stationary state have been found to be close to each other, with a slightly larger region of homogeneity, on average, for the present facility. This region proves to be smaller in the direction joining the subwoofers $(40 \mathrm{~mm})$ than in the direction of the bisectors between the subwoofers $(60 \mathrm{~mm})$. This shows that the size of the homogeneity domain is determined by the depth of mutual penetration of the facing jets. It constitutes the main limitation of this technique for generating turbulence. Increasing the size seems difficult and our attempt to use large synthetic jets to reach this objective is a posteriori not a very efficient solution. The mean flow velocity, the rms turbulence fluctuations and the integral length scale corresponding to the Reynolds number investigated, are of the order of $0.04 \mathrm{~m} / \mathrm{s}, 0.9 \mathrm{~m} / \mathrm{s}$ and $40 \mathrm{~mm}$ respectively.

Measurements in the naturally decaying turbulence created by stopping the subwoofers have also been performed. The decay follows a power law of the form $\overline{q^{2}} \propto t^{-n}$, with an exponent $\mathrm{n}=1.6$ which is slightly smaller than the value $\mathrm{n}=1.86$ found by Hwang and Eaton. This decay is faster than in isotropic grid turbulence, which is the mark of a spatially diffusing turbulence. 


\section{Acknowledgements}

The authors acknowledge the CNRS (Centre National de la Recherche Scientifique) and the French Ministry of Research and Technology (for their financial support. They express their gratitude to M. Massot for his scientific collaboration. They also acknowledge N. Grosjean and E. Jondeau for their efficient technical assistance in the handling of measuring techniques.

\section{References}

Batchelor GK (1953) The theory of homogeneous turbulence. Cambridge University Press.

Belmabrouk H, Michard M (1998) Taylor length scale measurement by laser Doppler velocimetry. Exp Fluids 25: 69-76.

Béra JC, Michard M, Grosjean N, Comte-Bellot G (2001) Flow analysis of two dimensional pulsed jets by particle image velocimetry. Exp Fluids 31:519-532.

Birouk M, Chauveau C, Sahr B, Quilgars A, Gokalp I (1996). Turbulence effects on the vaporization of monocomponent single droplets. Combustion Science and Tech. 113, 413428.

George, W.K.Jr, 1978. Processing of random signals. Proc. Dyn. Flow Conf., Tonsbakken, Denmark, 20-63.

Bos WJT, Shao L, Bertoglio JP (2007) Spectral imbalance and the normalized dissipation rate of turbulence. Phys Fluids 19: 045101.

Burattini P, Lavoie P, Antonia RA (2005). On the normalized turbulent energy dissipation rate. Phys Fluids 17: 098103.

Comte-Bellot G, Corrsin S (1966). The use of a contraction to improve the isotropy of gridgenerated turbulence. J Fluid Mech 25: 657-682.

De Jong J, Cao L, Woodward SH, Salazar JPLC, Collins LR, Meng H (2009) Dissipation rate estimation from PIV in zero-mean isotropic turbulence. Exp Fluids 46: 499-515. 
De Silva IPD, Fernando HJS (1994) Oscillating grids as a source of nearly isotropic turbulence. Phys Fluids, 6: 2455-2464.

Douady S, Couder Y, Brachet M E (1991) Direct observation of the intermittency of intense vorticity filaments in turbulence. Phys Rev Lett 67: 983-986.

Friedman PD, Katz J (2002) Mean rise rate of droplets in isotropic turbulence. Phys Fluids 14: $3059-3073$.

Glezer A, Amitay M (2002) Synthetic jets. Annu Rev Fluid Mech 34: 503-529.

George WK Jr (1978) Processing of random signals. Proc. Dyn. Flow Conf., Tonsbakken, Denmark, 20-63.

Hwang W, Eaton JK (2001) A new facility for study of attenuation of homogeneous isotropic gas turbulence by solid particles. $4^{\text {th }}$ International Conference on Multiphase Flow. New-Orleans. CD-rom, paper 125.

Hwang W, Eaton JK (2004a) Creating homogeneous and isotropic turbulence without a mean flow. Exp Fluids 36: 444-454.

Hwang W, Eaton JK (2004b) Modification of homogeneous and isotropic turbulence by solid particles. Tech Rep TF-90. Stanford University.

Hwang W, Eaton JK (2006) Homogeneous and isotropic turbulence modulation by small heavy (St 50) particles. J Fluid Mech 564: 361-393.

Kolmogorov AN (1941) The local structure of turbulence in an incompressible viscous fluid for very large Reynolds numbers. Dokl. Akad. Nauk SSSR 30: 299-303.

Liu S, Katz J, Meneveau C (1999) Evolution and modelling of subgrid scales during rapid straining of turbulence. J Fluid Mech 387 : 281-320.

Pope SB (2000) Turbulent Flows. Cambridge University Press, Cambridge. 
Sheng J, Meng H, Fox R (2000) A large eddy PIV method for turbulence dissipation rate estimation. Chem Eng Sci 55: 4423-4434.

Smith,BL, Glezer A (1998) The formation and evolution of synthetic jets. Phys Fluids 10: 2281-2297.

Sreenivasan KR (1998) An update on the energy dissipation rate in isotropic turbulence. Phys Fluids 10: 528-529.

Tennekes H (1975) Eulerian and Lagrangian time microscales in isotropic turbulence. J Fluid Mech 67: 561-567.

Tennekes H, Lumley JL (1974) A first course in turbulence. MIT Press, Cambridge, Mass, US. Third printing.

Uberoi MS, Wallis S (1967) Effect of grid geometry on turbulence decay. Phys Fluids 10: 1216-1223.

Variano EA, Cowen EA (2008) A random-jet-stirred turbulence tank. J Fluid.Mech 604: 1-32.

Villermaux E, Hopfinger EJ (1994) Periodically arranged co-flowing jets. J Fluid Mech 263: 63-92.

Yeung PK, Sawford BL (2002) Random-sweeping hypothesis for passive scalars in isotropic turbulence. J Fluid Mech 459: 129-138. 

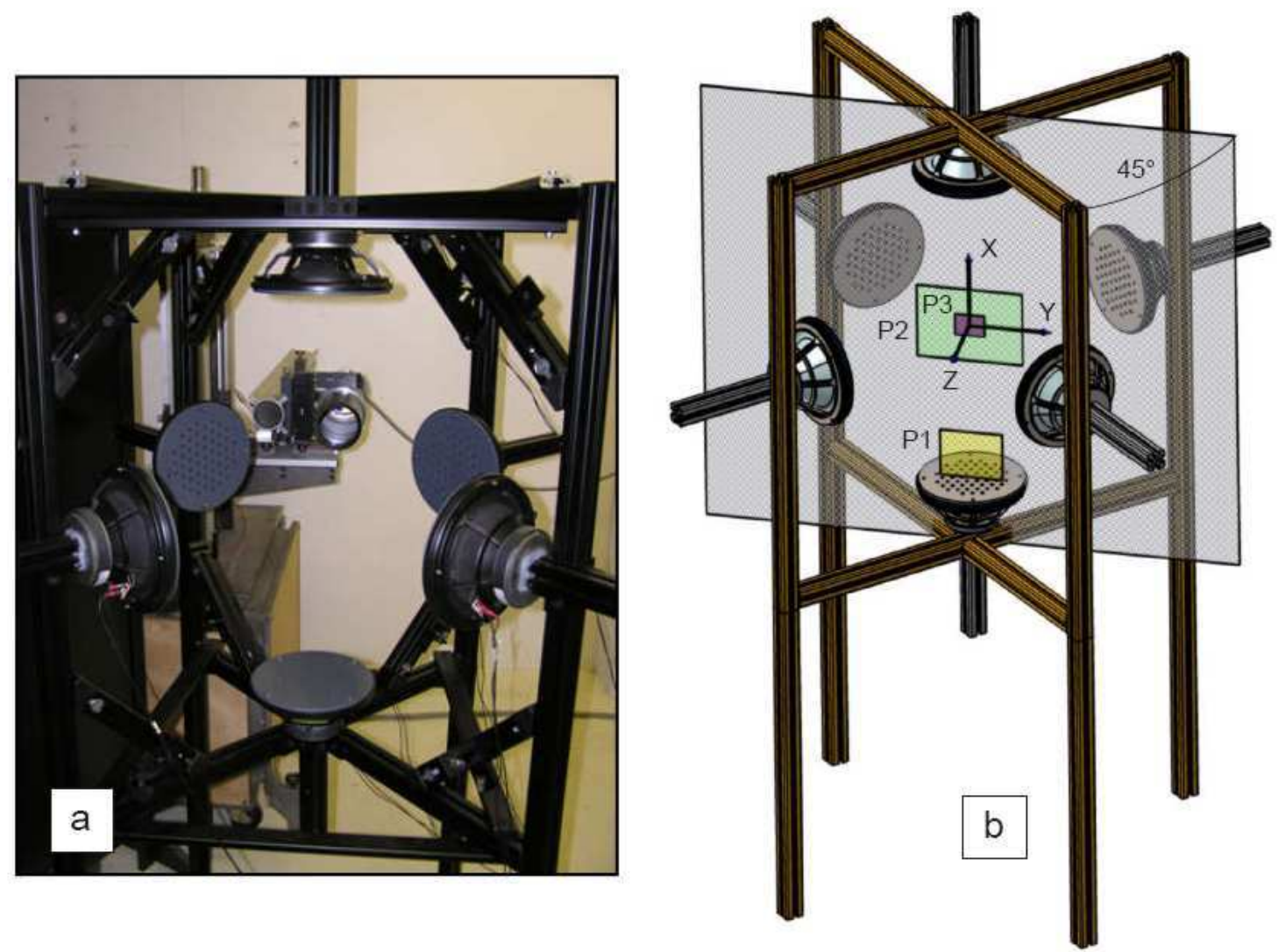

Fig.1 a: Experimental facility; b: sketch of the facility. P1:130 x $103 \mathrm{~mm}$; P2: $162 \times 219 \mathrm{~mm}$; P3: $62 \times 46 \mathrm{~mm}$.

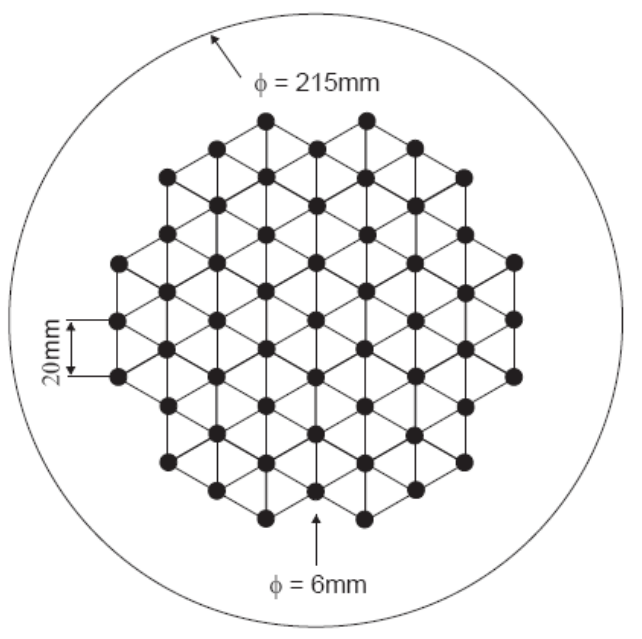

Fig.2: Schematic of the hole pattern. 

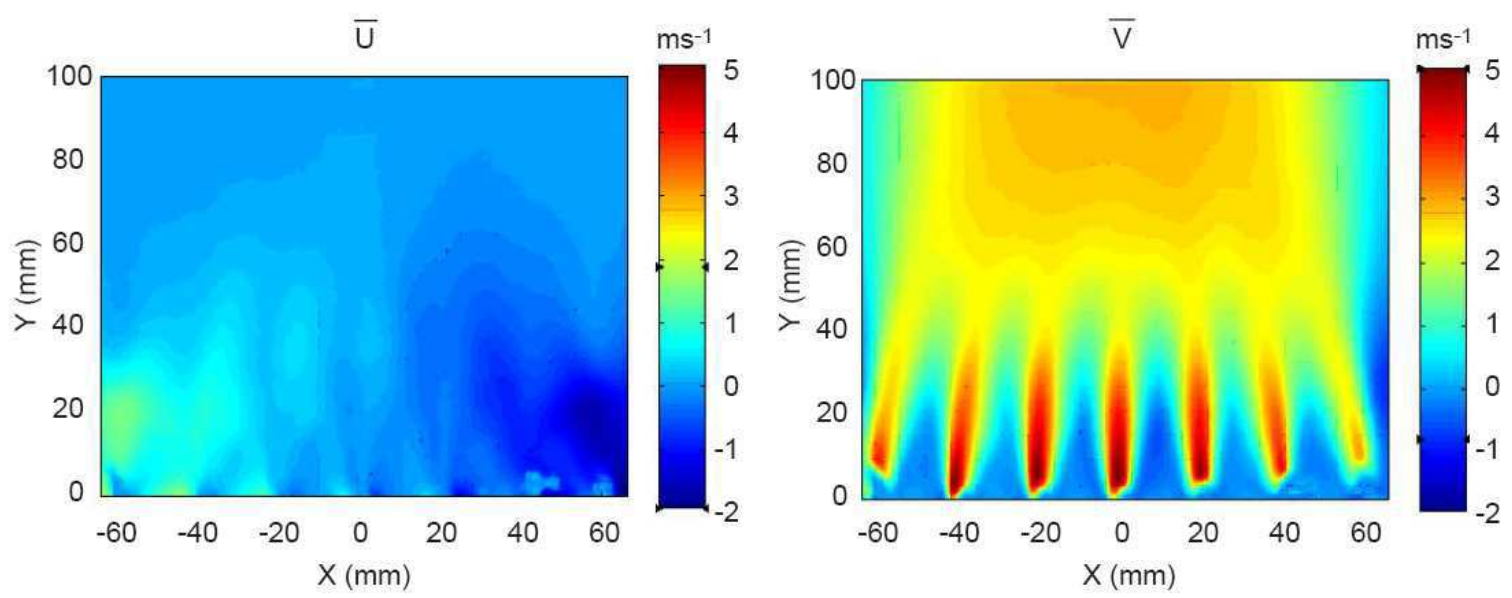

Fig.3 Mean velocity field behind the perforated cap of the speakers (plane P1).
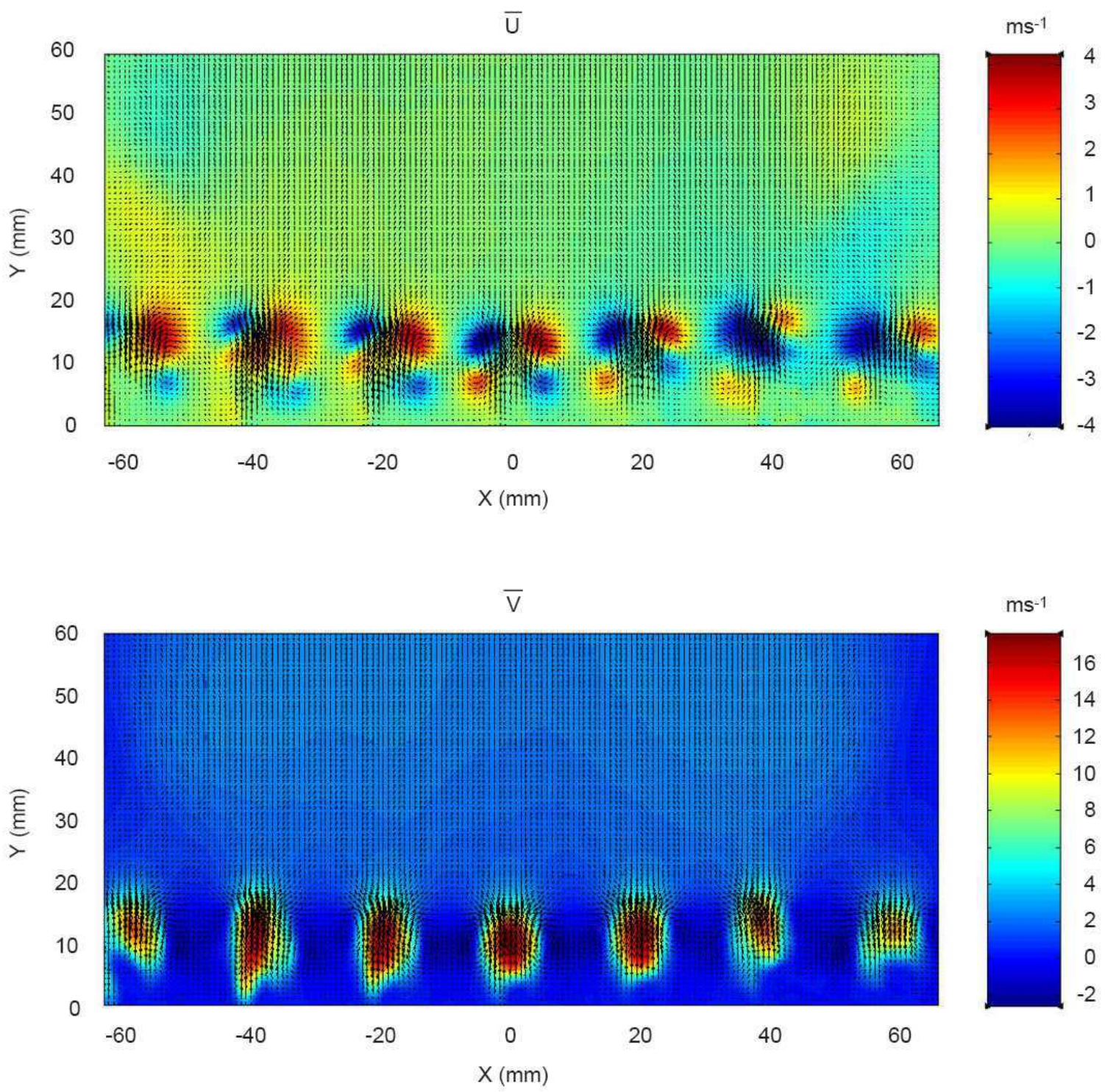

Fig.4 Phase-averaged velocity field at the beginning of the blowing phase (plane P1). 

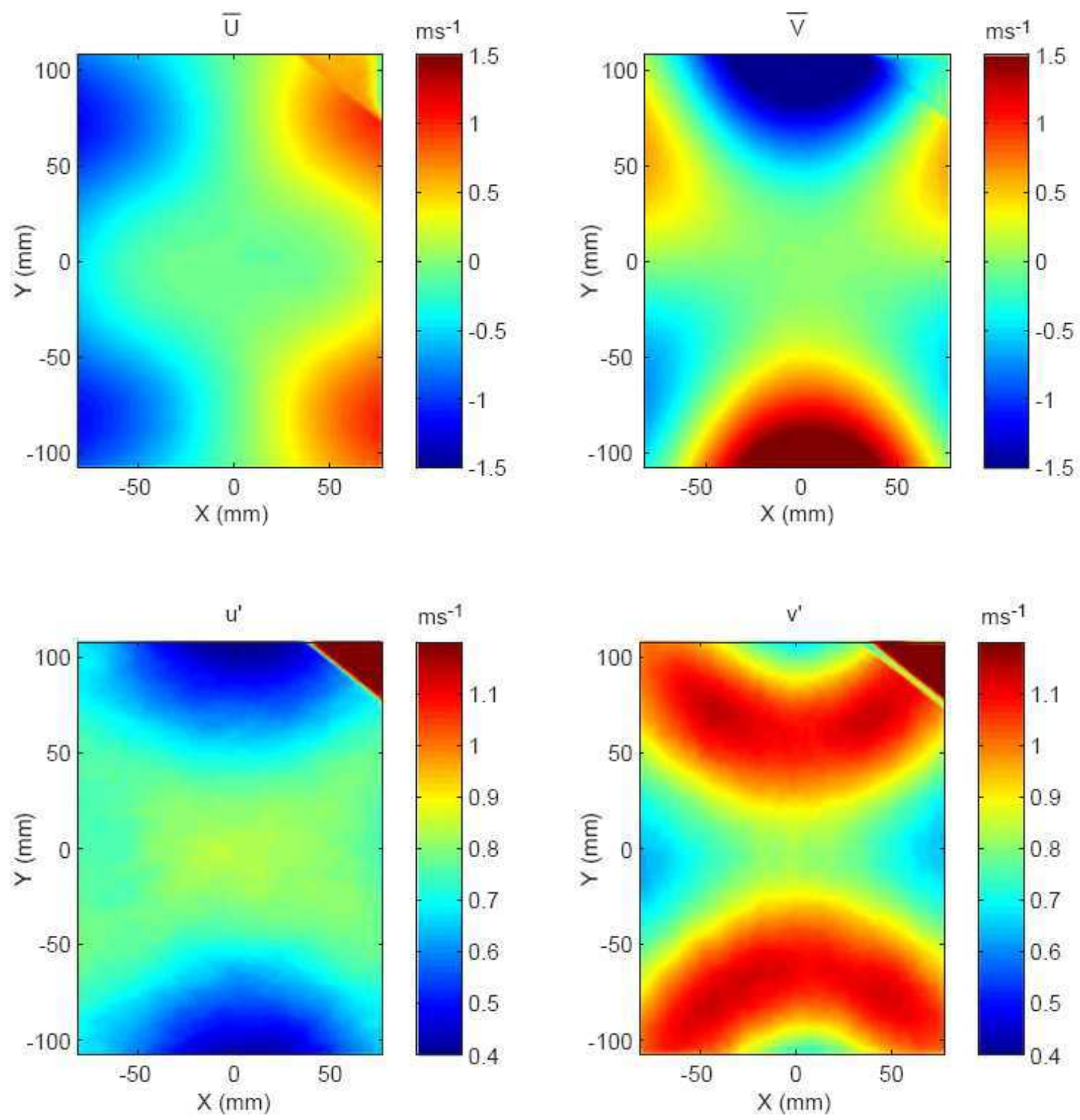

Fig.5 Maps of mean velocity and RMS velocity fluctuations in the large field of view (plane P2). 

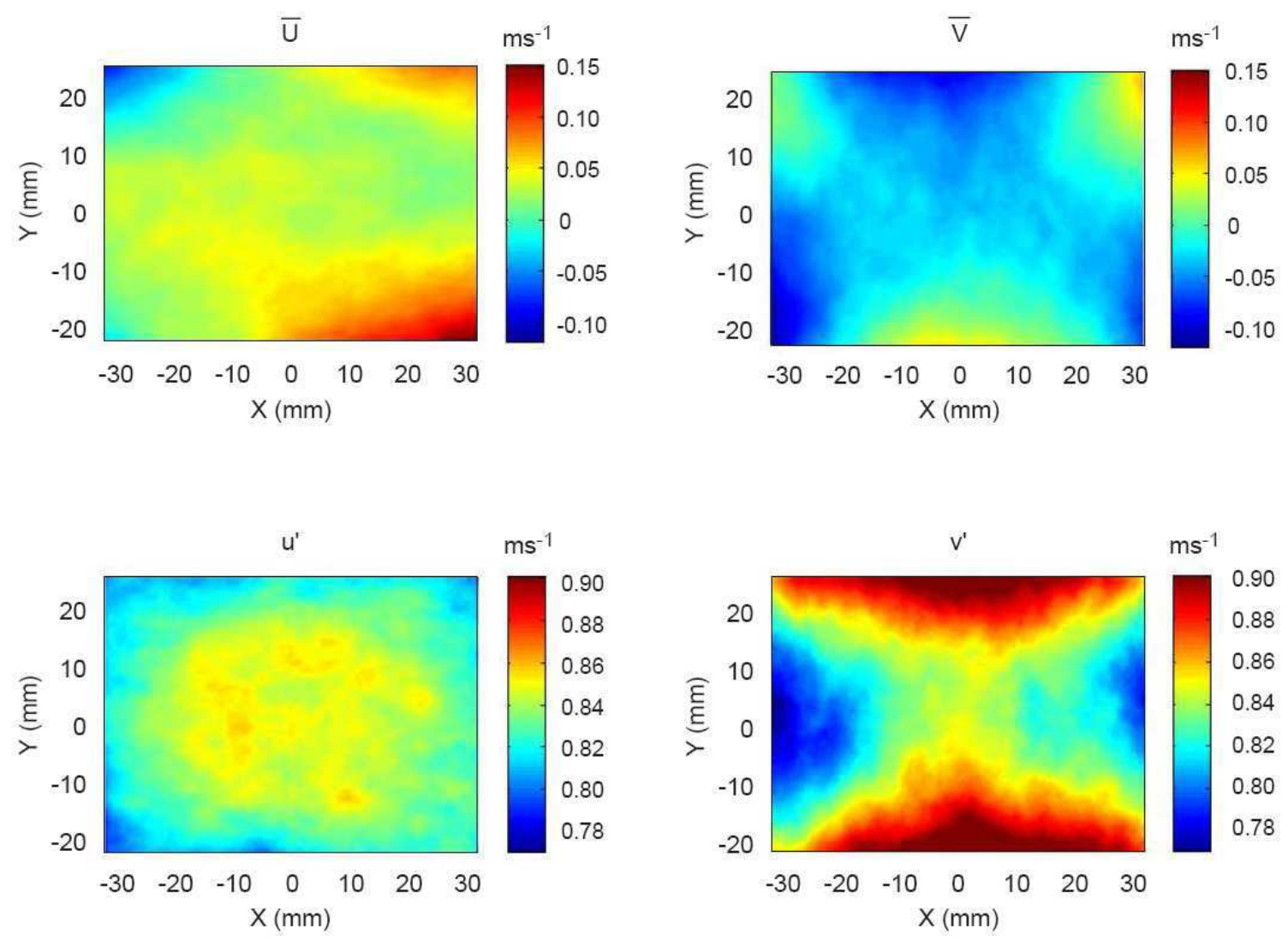

Fig.6 Maps of mean velocity and RMS velocity fluctuations (plane P3). 

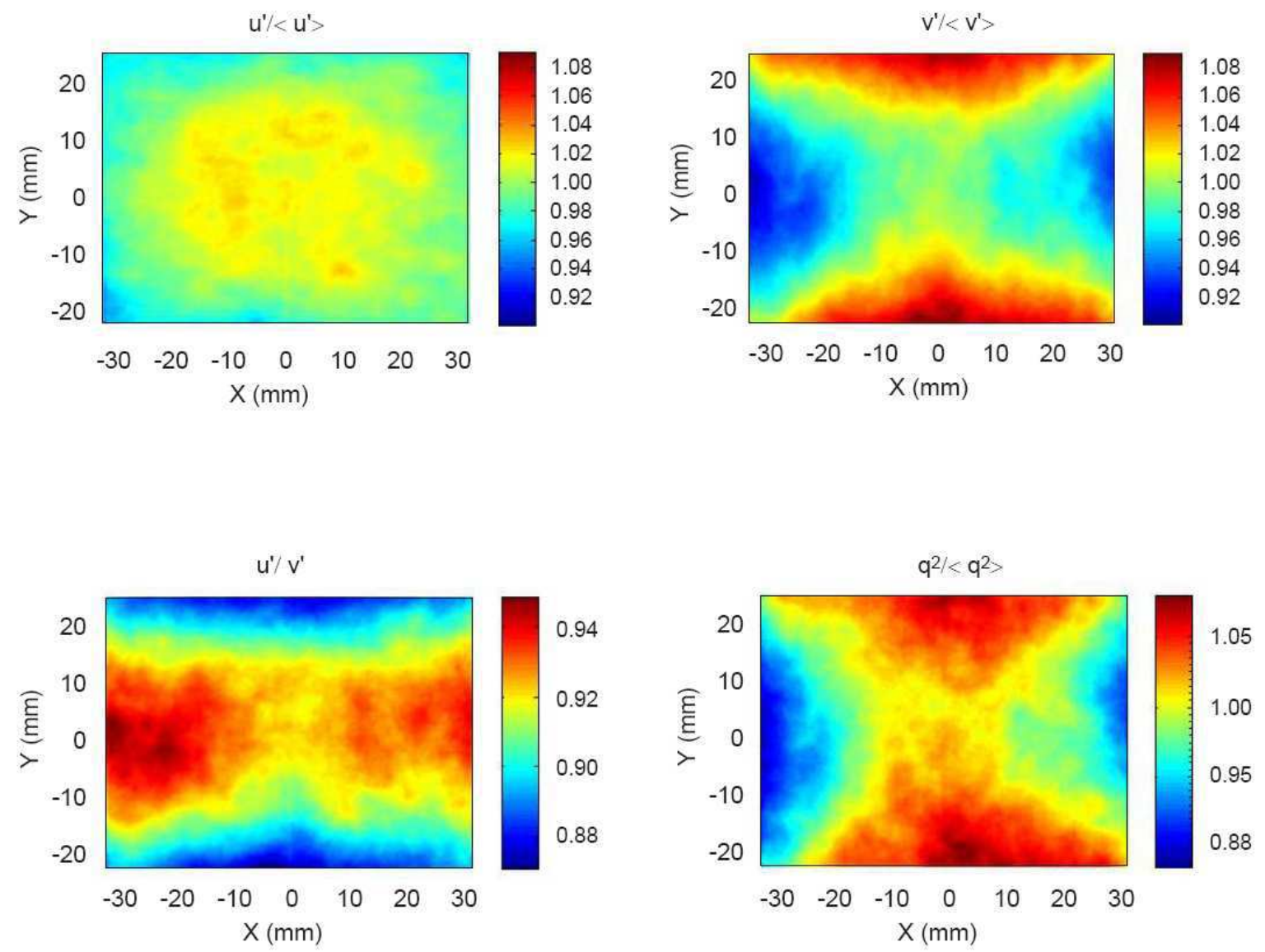

Fig.7 Maps of non-dimensional RMS velocity fluctuations, isotropy ratio and non-dimensional kinetic energy, inside the turbulence box.
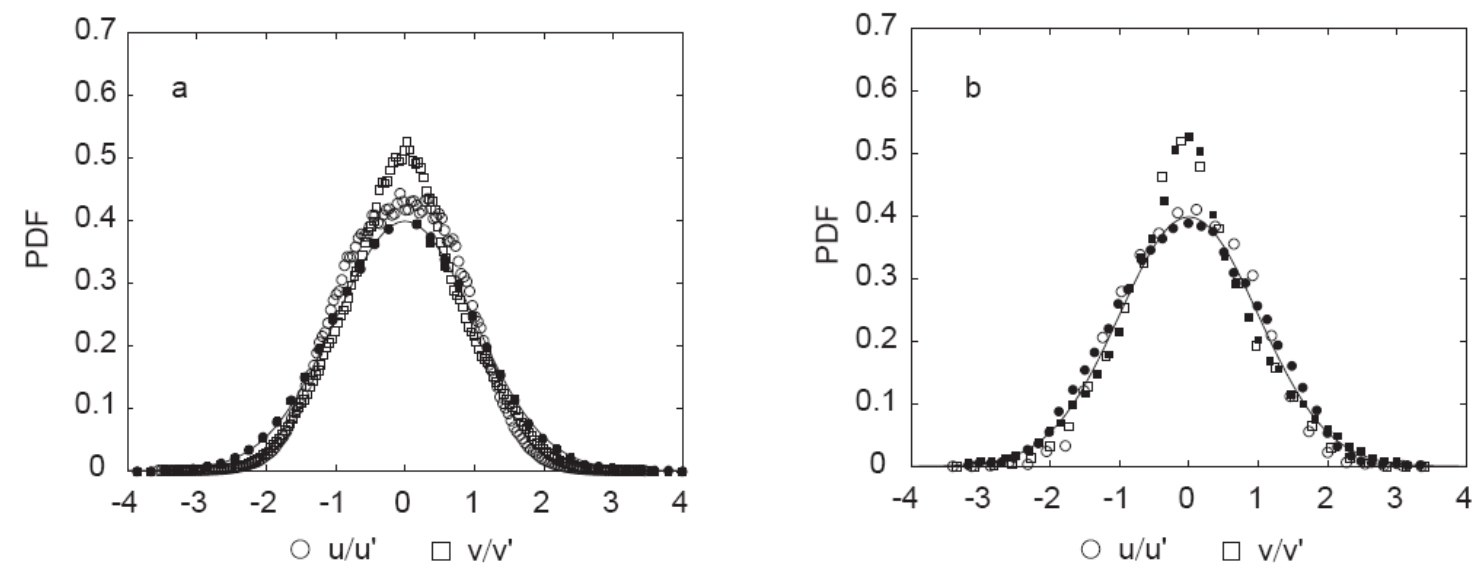

Fig. 8 PDFs of non-dimensional velocity fluctuations. a: measured by PIV at all locations (open symbols) compared with the PDFs by Hwang and Eaton (2004) (closed symbols), _ Gaussian PDF; b: measured by PIV (open symbols) and LDA (closed symbols) at the centre. 


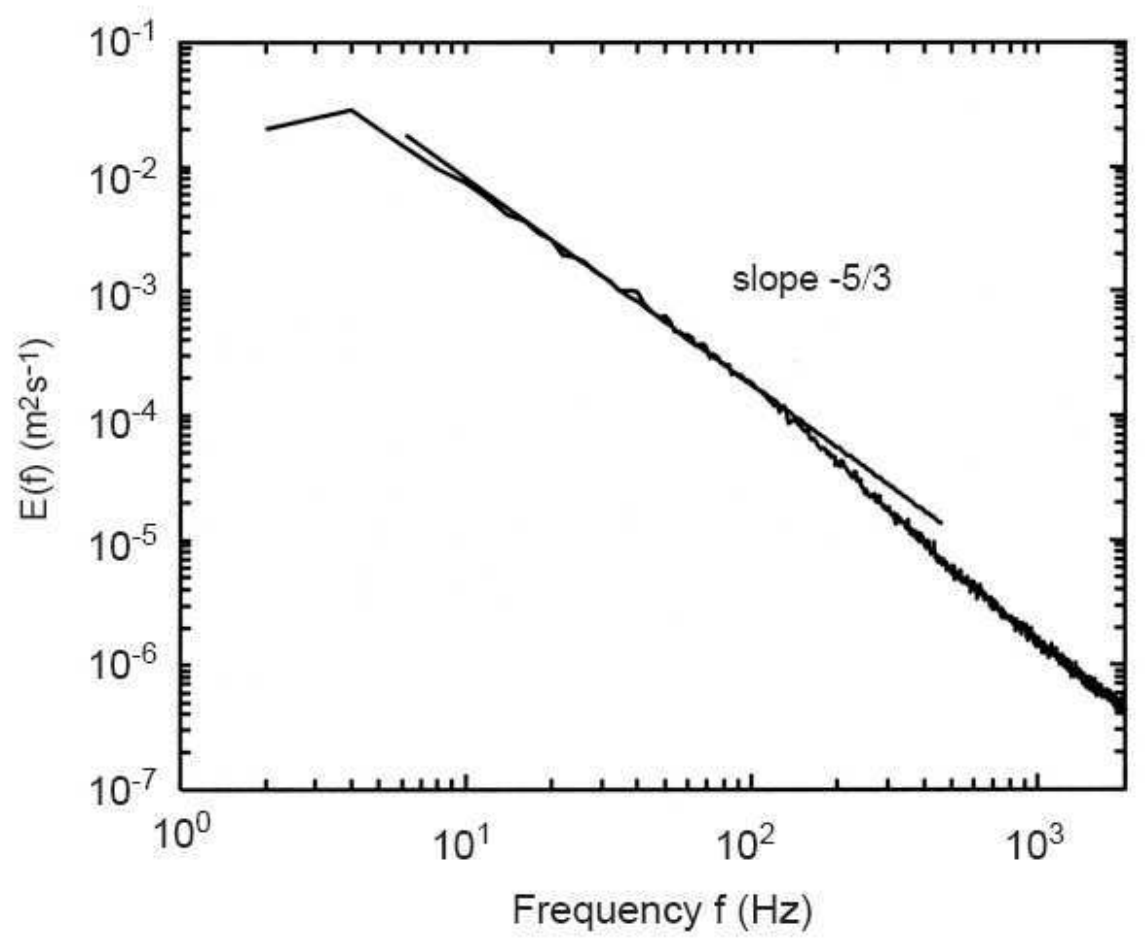

Fig.9 One-dimensional turbulent kinetic energy Eulerian frequency spectrum.

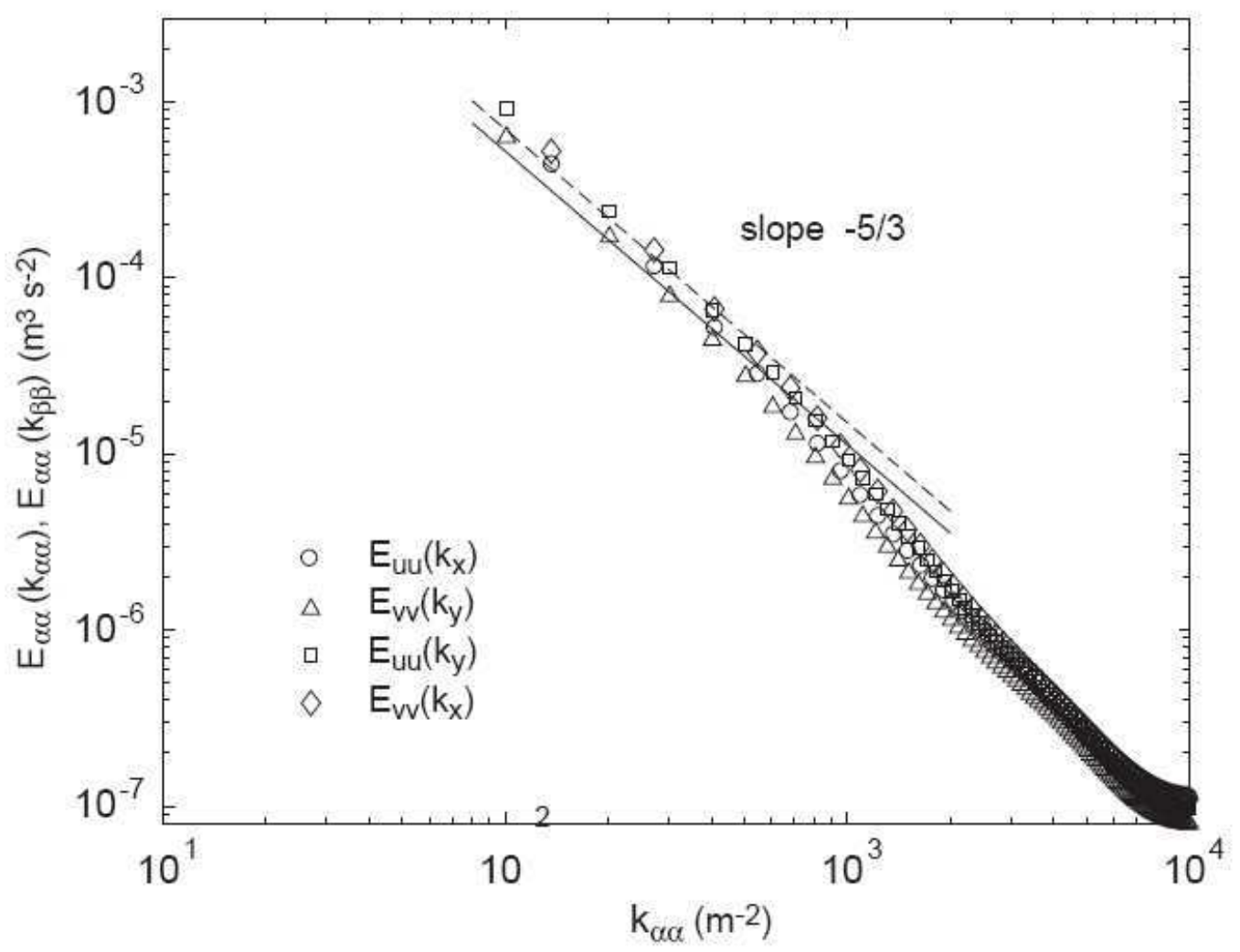

Fig.10 Longitudinal and transverse one-dimensional spatial energy spectra, as measured by PIV. Straight and dashed lines: Kolmogorov's model eq. [1,2]. 


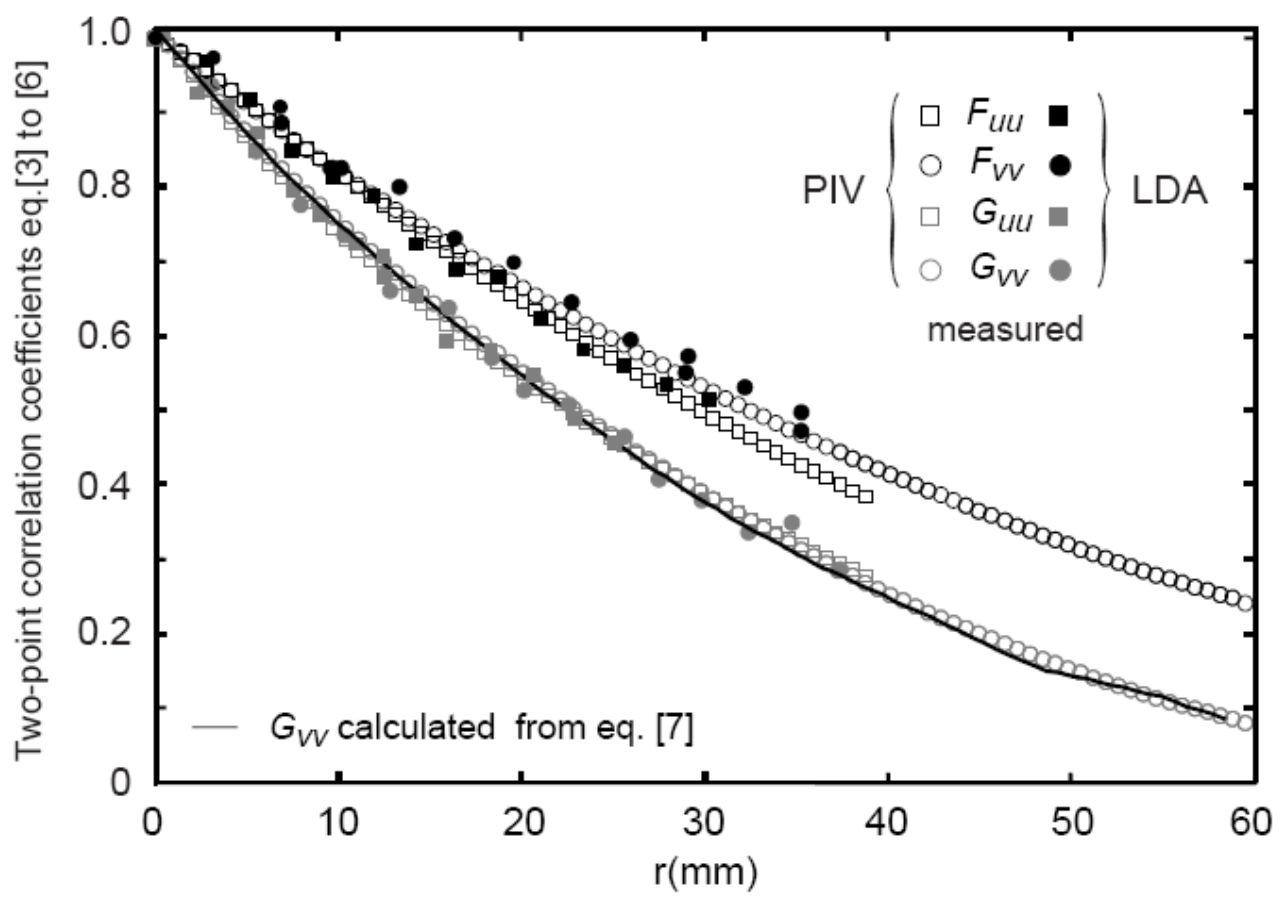

Fig.11 Relationship between the longitudinal and lateral velocity correlations. PIV: coefficients spatially averaged over the domain of homogeneity $(60 \times 40 \mathrm{~mm})$; LDA: coefficients averaged at $\pm r$ along the $X$ and $Y$ axis.

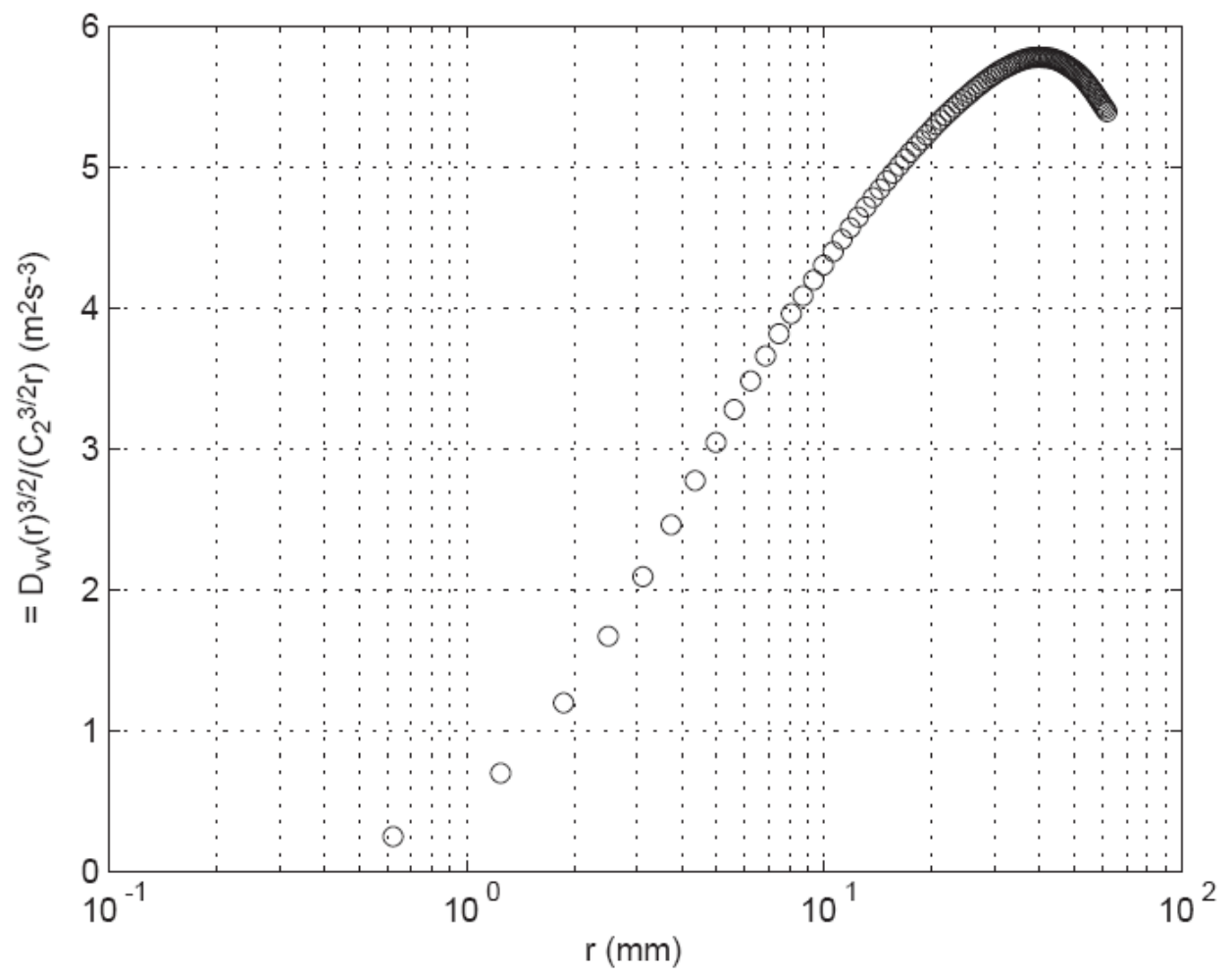

Fig.12 The second-order longitudinal velocity structure function plotted under the usual compensated form to determine the dissipation rate. $C_{2}=2$, eq. [11] 


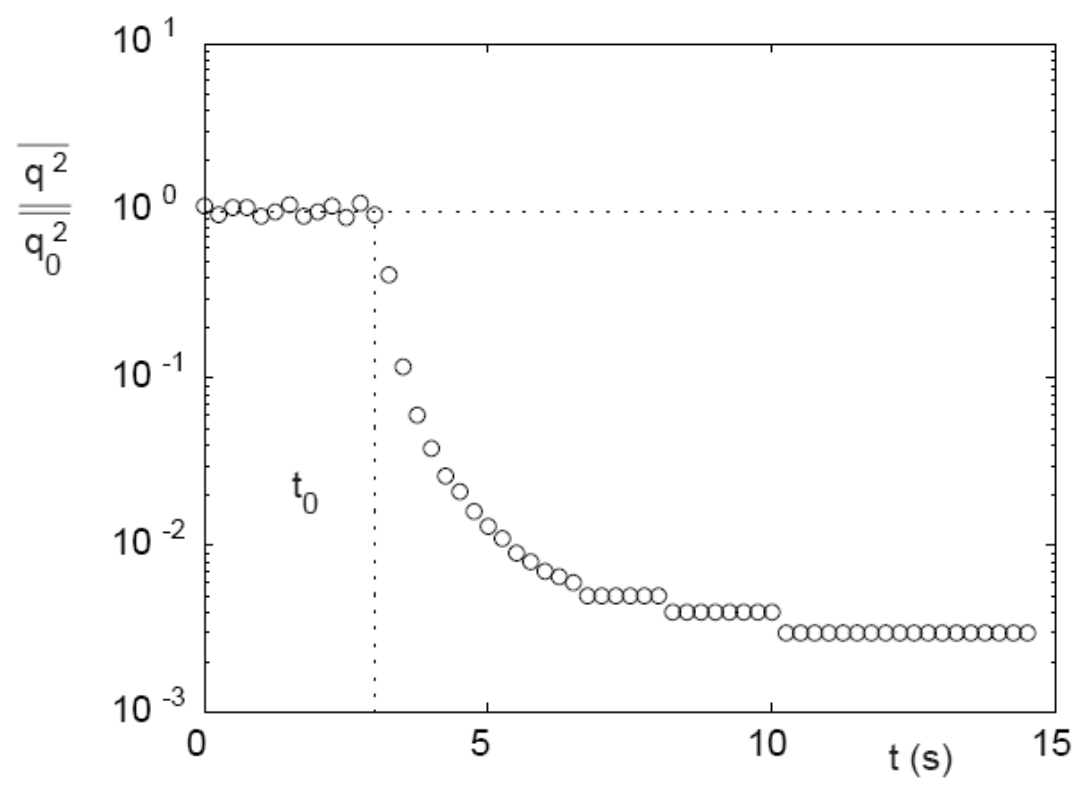

Fig.13 Decay of the turbulent kinetic energy $q^{2}$ with time, normalized by the energy $\mathrm{q}_{0}^{2}$ at time $\mathrm{t}_{0}$ when forcing is stopped.

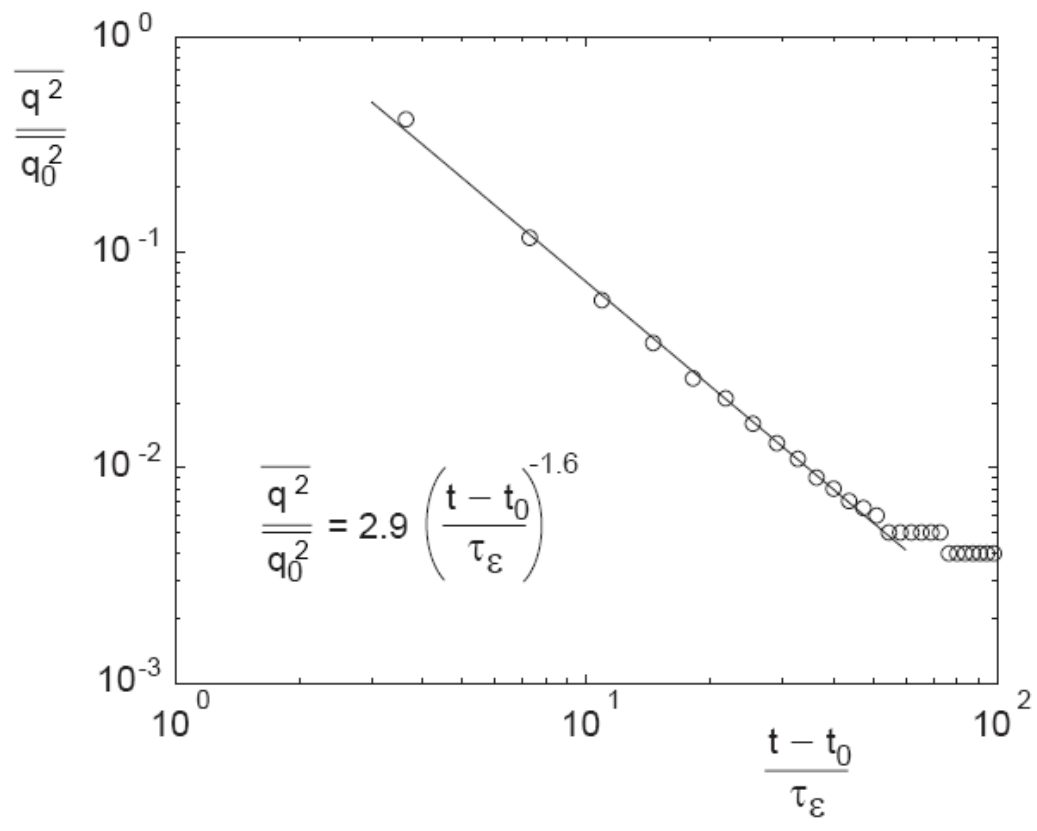

Fig.14 Decay of the normalized turbulent kinetic energy $\overline{q^{2}} / \overline{q_{0}^{2}}$ in log-log coordinates. Time is normalized by the eddy turnover time $\tau_{\varepsilon}$. 